\title{
Pouvoirs publics, populations amérindiennes et bushinenge en Guyane Française
}

Quelles relations depuis la décentralisation (1982-2008)?

Authorities and populations of American Indians and bushinenge in French

Guiana: what has become the relationship since the decentralization (1982-2008)

\section{Maude Elfort}

\section{OpenEdition}

\section{Journals}

Édition électronique

URL : http://journals.openedition.org/plc/819

DOI : $10.4000 /$ plc. 819

ISSN : 2117-5209

\section{Éditeur}

L'Harmattan

\section{Édition imprimée}

Date de publication : 1 janvier 2010

Pagination : 67-92

ISBN : 978-2-296-1141-3

ISSN : 1279-8657

Référence électronique

Maude Elfort, "Pouvoirs publics, populations amérindiennes et bushinenge en Guyane Française » Pouvoirs dans la Caraïbe [En ligne], 16 | 2010, mis en ligne le 22 septembre 2011, consulté le 01 mai 2019. URL : http://journals.openedition.org/plc/819; DOI : 10.4000/plc.819 


\title{
POUVOIRS PUBLICS, POPULATIONS AMERINDIENNES ET BUSHINENGE EN GUYANE FRANÇAISE : \\ QUELLES RELATIONS DEPUIS LA DECENTRALISATION (1982-2008) ?
}

\author{
Maude ELFORT \\ Maître de conférences en Droit public \\ Membre du CRPLC \\ Université des Antilles et de la Guyane
}

Soumise depuis le $\mathrm{XV}^{\mathrm{e}}$ siècle à la colonisation, érigée en département par la loi du 19 mars $1946^{1}$, la Guyane ${ }^{2}$ présente une différence essentielle avec les trois autres départements d'outre-mer ${ }^{3}$ : la présence sur son sol d'une population autochtone d'origine ${ }^{4}$ et d'une population bushinenge issue de l'esclavage ${ }^{5}$.

Les relations entre l'administration française et ces populations sont anciennes, elles remontent à la période coloniale. Longtemps l'isolement des

\footnotetext{
${ }^{1}$ J.O., Lois et décrets, 20 mars 1946, p. 2294.

2 Située à proximité de l'Equateur, la Guyane partage des frontières communes avec le Surinam et le Brésil. A l'inverse des autres départements d'outre-mer constitués d'îles et caractérisés par de faibles superficies et des ressources naturelles limitées, la Guyane fait partie intégrante du continent sud-américain, dispose d'une superficie importante $\left(83534 \mathrm{~km}^{2}\right.$ dont 7618100 ha de surface boisée soit $91 \%$ du territoire) et bénéficie d'un potentiel naturel important en matière forestière et halieutique. La démographie de la Guyane présente à la fois une forte croissance et une structure en déséquilibre : de 1999 à 2006, la population guyanaise a augmenté de $202 \%$ (dix fois plus vite qu'en Métropole), le taux de progression annuel s'élevant à 3,8\% par an ; le dernier recensement de 2006 (TER/INSEE, 2007/08) évalue la population à 201996 habitants. La répartition de la population par tranche d'âge révèle que les moins de 20 ans représentent près de la moitié de la population alors que les plus de 60 ans, seulement $6 \%$. Le déséquilibre est aussi territorial : plus de la moitié de la population réside dans «l'île» de Cayenne, la capitale qui, à elle seule, rassemble un tiers de la population totale.

${ }^{3}$ Guadeloupe, Martinique, Réunion.

${ }^{4}$ Les populations amérindiennes autochtones - peu nombreuses (5 500 en 2003) environ $4 \%$ de la population - se répartissent entre six groupes linguistiques (Galibi, Arawak, Palikur, Wayana, Emerillon, Wayampi) et deux zones géographiques : les Amérindiens du littoral, majoritairement acculturés et utilisant le français et le créole comme langues véhiculaires, et les Amérindiens de l'Intérieur dispersés le long des fleuves en petites communautés perpétuant les modes de vie traditionnels.

${ }^{5}$ Les Bushinenge sont les descendants d'esclaves révoltés qui au XVIII ${ }^{\mathrm{e}}$ siècle, ont fui les plantations du Surinam pour s'installer en Guyane, le long des fleuves. Nombreux (environ 37200 en 2003), ils sont répartis en quatre groupes linguistiques (Saramaka, Ndyuka, Aluku, Paramaka) et deux zones géographiques : le littoral et l'Intérieur. Ces communautés, inégalement acculturées, perpétuent, elles aussi, les modes de vie traditionnels.
} 
Amérindiens et des Bushinenge et leur faible importance statistique s'accompagnent de l'absence de reconnaissance juridique. En 1930, le principe de respect des modes de vie amérindiens et bushinenge, invoqué par l'Etat, détermine la mise en place d'une structure administrative autonome, placée sous l'autorité directe du gouverneur de la Guyane : le territoire de l'Inini ${ }^{6}$.

La fin des années soixante marque une rupture avec l'attribution aux Amérindiens et aux Bushinenge de la citoyenneté française ${ }^{7}$ par la mise en place d'un programme de francisation intensif et la municipalisation du territoire de l'Inini ${ }^{8}$.

Dès 1970, la nécessité de protéger les populations amérindiennes incite le préfet à réglementer l'accès du tiers sud de la Guyane 9 .

En 1984, année importante, se tient à Awala le premier rassemblement des Amérindiens de Guyane, organisé par l'Association des Amérindiens de Guyane Française (AAGF). Les participants affirment leur «volonté de prendre place dans l'espace politique local ${ }^{10}$. Une dynamique est lancée. Elle va se renforcer avec la création de la Fédération des Organisations Amérindiennes de Guyane $(\mathrm{FOAG})^{11}$. Simultanément les travaux des anthropologues et des ethnologues sur les effets négatifs de la politique de francisation et d'assimilation forcée conduisent à réfléchir sur des pédagogies mieux adaptées à la situation particulière des populations amérindiennes. La participation de représentants amérindiens ${ }^{12}$, à partir de 1996, aux sessions annuelles du groupe de travail de l'Organisation des

\footnotetext{
${ }^{6}$ Sur ce point, voir I. Arnoux « Des autochtones dans la région ultra-périphérique de Guyane. Et alors ? », Droit et Cultures, Paris, l'Harmattan, 1999 ; M. Elfort, « Les communes rurales de Guyane », Bulletin de la société d'histoire de la Guadeloupe, $\mathrm{n}^{\circ}$ 150/151, déc. 2008.

${ }^{7}$ Qui s'accompagne d'un programme de francisation intensif.

${ }^{8}$ Cinq communes sont créées : Grand-Santi, Saint-Elie, Saûl, Camopi et Maripasoula.

${ }^{9}$ Commune de Camopi et des environs. Arrêté préfectoral du 14/09/1970, modifié par l'arrêté du 26/7/1978.

${ }^{10}$ Collomb Gérard et Félix Tiouka. Na'na Kali'na : une histoire des Kali'na en Guyane, Ibis Rouge Editions, 2000, p. 121.

${ }^{11}$ En 1992, elle succède à l'AAGF.

${ }^{12}$ A partir de 1996.
} 
Nations Unies (ONU) sur les peuples autochtones, les incitent à s'interroger sur les possibilités d'expression juridique de leurs particularismes ${ }^{13}$.

Parallèlement, les années quatre-vingt voient la mise en place de la décentralisation : communes, départements et régions reçoivent d'importantes compétences dans les domaines de l'urbanisme, de l'aménagement du territoire, du cadre de vie, de l'environnement ou de l'identité culturelle ${ }^{14}$. Dans ce nouveau cadre juridique, les collectivités territoriales sont appelées à établir des relations avec les populations amérindiennes et bushinenge.

Jusqu'en 2006, face aux revendications identitaires de ces communautés, l'Etat et les collectivités répondent par des mesures diverses et ponctuelles. Celles-ci permettent la prise en compte implicite de traditions et coutumes officiellement ignorées du droit étatique.

Un nouveau tournant s'amorce en 2003 avec la réforme constitutionnelle du 28 mars. Désormais, unité ne se confond plus avec uniformité : l'heure est à la diversité statutaire et à la reconnaissance des particularismes. En septembre 2007, la France signe la Déclaration sur les droits des peuples autochtones qui énumère les droits (nombreux) reconnus par l'Organisation des Nations Unies aux autochtones ${ }^{15}$; la réforme constitutionnelle de juillet 2008 affirme que les langues régionales appartiennent au patrimoine de la France (art. 75-1 de la Constitution).

Les facteurs d'évolution ne sont pas seulement juridiques, ils sont aussi d'ordre démographique et sociologique : en 1986, au lendemain de la guerre civile entre les Marrons et l'armée nationale du Suriname, des milliers de Bushinenge émigrent en Guyane et nombre d'entre eux y restent après la fin du conflit en 1992. En 2003 ces derniers sont estimés au nombre

\footnotetext{
${ }^{13}$ Sur l'histoire des Amérindiens en Guyane, cf. J. Hurault. «Français et Indiens en Guyane, 1604-1972 ». Paris : UGE (10/18), 1972. Egalement, G.Collomb, F. Tiouka. Na'na Kali'na. Ibis Rouge Editions, 2000 et les travaux de P.et F. Grenand. Les populations amérindiennes de Guyane, Atlas de la Guyane, CNRS/ORSTOM, Paris, 1979. Paradoxalement, les Bushinenge semblent peu concernés par ces questions identitaires.

${ }^{14}$ Loi $\mathrm{n}^{\circ} 82-213$ du 2 mars 1982 relative aux droits et libertés des communes, départements et régions. J.O du 3 mars, p. 730 ; loi n 83-8 du 7 janvier 1983 et loi ${ }^{\circ} 83-663$ du 22 juillet 1983 relative à la répartition des compétences entre communes, départements, régions et Etat, J.O.du 9 janvier, p. 215 et J.O. du 23 juillet, p. 2286.

${ }^{15}$ Nations Unies, $108^{\mathrm{e}}$ séance plénière, jeudi 13/9/07, A/61/PV.108.
} 
de 37200 , soit plus de $20 \%$ de la population ${ }^{16}$. Incontestablement, la progression démographique des populations amérindiennes constitue un facteur favorable pour la crédibilité et la légitimité de leur action. De façon logique, ces groupes s'organisent socialement et politiquement : ils revendiquent le droit de participer aux décisions les concernant et le respect de leurs spécificités culturelles. Parallèlement, on observe depuis une dizaine d'années une évolution des mentalités dans la société guyanaise. En témoigne significativement le document d'orientation de la Commission mixte département/région appelant les peuples autochtones à participer à une «identité guyanaise multiculturelle », aux côtés des autres communautés de base, créoles et noirs-marrons, et à " une communauté de destin «intégrant les différents groupes ethniques ${ }^{17}$. Une telle conjonction explique, sans doute, la mutation des rapports pouvoirs publics/communautés autochtones et noires-marrons : à la reconnaissance implicite succède désormais la reconnaissance explicite des particularismes.

\section{I - La reconnaissance implicite des particularismes coutumiers}

Cette reconnaissance implicite est perceptible à travers, d'une part, les mesures élaborées par l'Etat, et les pratiques des collectivités territoriales, d'autre part.

\section{A - Les mesures étatiques}

Les politiques étatiques à l'égard des Amérindiens et des Bushinenge s'inscrivent dans un régime juridique traditionnellement construit en France sur le principe d'interdiction des distinctions fondées sur des critères ethniques religieux ou raciaux (article 1er de la Constitution). Sur la scène internationale, ce principe conduit la France à dénier aux groupes identifiés par des particularismes ethniques, linguistiques ou religieux, toute reconnaissance juridique ${ }^{18}$. En droit interne, il explique le

\footnotetext{
${ }^{16}$ R. et S. Price. Les Marrons. Châteauneuf-le-Rouge (France) : Vents d'ailleurs, 2003.

${ }^{17}$ Document d'orientation d'un Pacte de développement pour la Guyane, novembre 1998. Commission mixte département/région.

${ }^{18}$ Cf. B. de Witte, «Minorités nationales : reconnaissance et protection », Pouvoirs, 1991, $\mathrm{n}^{\circ} 57$, p. 116. L'auteur rappelle que la France avait...par une déclaration interprétative, exclu
} 
refus de toute reconnaissance constitutionnelle de minorités comme en témoigne la décision du Conseil Constitutionnel en $1991^{19}$. C'est ce même principe «d'unicité du peuple français » qui conduit le juge constitutionnel à s'opposer en $1996^{20}$ aux dispositions prévoyant l'enseignement obligatoire d'une langue régionale dans les écoles, et à déclarer en 1999 que la Charte européenne des langues régionales ou minoritaires comporte des clauses contraires à la Constitution ${ }^{21 .}$

Les politiques étatiques dépendent ensuite du régime constitutionnel et administratif de la collectivité concernée : la Guyane est un département d'outre-mer, soumis, en vertu de l'article 73, au principe d'identité législative, c'est à dire à l'applicabilité de plein droit des lois et règlements. Certes, le même article prévoit que « le régime législatif et l'organisation administrative des DOM peuvent faire l'objet de mesures d'adaptation nécessitées par leur situation particulière $»^{22}$. Cependant, on le sait, la portée de cette disposition a fait l'objet d'une interprétation restrictive par le Conseil Constitutionnel ${ }^{23 .}$ Elle explique sans doute le faible bilan dont peut se prévaloir l'Etat : on peut ainsi évoquer au titre de la reconnaissance des particularismes de ces populations :

- la création en 1984, à l'initiative du préfet, d'un Comité de coordination des actions en faveur des Amérindiens de la Guyane Française et des populations du Maroni ${ }^{24}$;

\footnotetext{
l'application de l'article 27 du Pacte des Nations Unies en territoire français, parce que la France se définissait dans la Constitution comme indivisible et ne pouvait donc reconnaitre l'existence de minorités.

${ }^{19}$ Décis. ${ }^{\circ}$ 91-290 DC du 9/5/91, Loi portant statut de la collectivité territoriale de Corse, Rec. p. 50 ; Grandes décisions.11e éd., $\mathrm{n}^{\circ}$ 44. Paris : Dalloz, 2001. En l'espèce, le juge estime que le concept de peuple Corse, composante du peuple français est inconstitutionnel au motif que la Constitution ne connaît qu'un seul peuple composé de tous les citoyens français sans distinction d'origine, de race ou de religion.

${ }^{20}$ Décis. $n^{\circ}$ 96-373 DC du 9/4/96 sur le statut d'autonomie de la Polynésie française, J.O.du 13 avril 1996, p. 5724.

${ }^{21}$ Décis. $n^{\circ}$ 99-412 DC du 15/6/99, Charte européenne des langues régionales ou minoritaires, Rec. p. 71.

${ }^{22}$ Il s'agit là de la formulation antérieure à la réforme constitutionnelle du 28 mars 2003.

${ }^{23}$ Décis. n $82-147$ DC du 2/12/1982 et décis. n 84-174 -DC du 25/7/1984.

${ }^{24}$ Qui ne s'est jamais réuni.
} 
- les décrets $\mathrm{n}^{\circ} 87-267$ du 14 avril $1987^{25}$ et $\mathrm{n}^{\circ}$ 92-46 du 16 janvier $1992^{26}$ relatifs aux concessions domaniales de l'Etat qui prévoient que les droits d'usage collectifs pour la pratique de la pêche et de la chasse des communautés d'habitants tirant traditionnellement leurs moyens de subsistance de la forêt sont reconnus par le préfet après avis des services fiscaux $^{27}$;

- l'élaboration par le Rectorat, en 1994, d'un Plan académique de développement des langues et cultures régionales ${ }^{28}$;

- l'institution de médiateurs culturels et de médiateurs bilingues ${ }^{29}$ ou encore l'intégration des arts plastiques et des arts du spectacle des Marrons et des Amérindiens au patrimoine culturel de la France.

Par ailleurs, dans les année quatre-vingt-dix, l'Etat crée trois nouvelles communes : Awala Yalimapo ${ }^{30}$, Grand Santi ${ }^{31}$, Papaichton ${ }^{32}$, en réponse aux demandes des populations concernées. Officiellement, ces créations ont été justifiées par la situation géographique des communes de Mana et de Grand-Santi-Papaichton, alors qu'en réalité elles reposaient sur des bases essentiellement ethniques. Cependant, une telle argumentation a permis d'échapper à la censure du juge, la jurisprudence admettant en effet, depuis longtemps, que des normes différentes puissent s'appliquer à des

\footnotetext{
25 J.O. du 16 avril 1987, p. 4316.

${ }^{26}$ J.O. du 17 février 1992, p. 811.

${ }^{27}$ Sur le contenu et la portée de ces décrets, cf. A. Calmont, «Le problème foncier en Guyane : le poids de la domanialité in La question de la terre dans les colonies et départements français d'Amérique. Paris, Karthala, 2000, p. 203.

${ }^{28}$ Cf. sur ce point, Décis. n ${ }^{\circ}$ 91-290 DC du 9 mai 1991, statut de la Corse, J.O., 14/5/91. En l'espèce, le Conseil affirme «que le fait pour le législateur d'autoriser la collectivité territoriale de la Corse à promouvoir l'enseignement de la langue et de la culture corse ne saurait être regardée comme portant atteinte à aucun principe de valeur constitutionnelle ».

${ }^{29}$ En 1998, 27 aides-éducateurs amérindiens et bushinenge ont été recrutés par l'Education Nationale sur le critère du bilinguisme ; ils interviennent principalement à Awala-Yalimapo et dans certaines écoles à majorité bushinenge de Saint Laurent du Maroni. A ce titre ils ont reçu une formation linguistique et pédagogique à l'Institut Universitaire de Formation des Maîtres grâce a un partenariat entre l'Institut Régional de Développement et l'Education Nationale.

${ }^{30}$ Créée en 1989 et peuplée en majorité de Kali'na.

${ }^{31}$ Créée en 1993, peuplée en majorité de Ndjuka.

${ }^{32}$ En 1993, peuplée en majorité d'Aluku.
} 
situations différentes ${ }^{33}$. En d'autres termes, un traitement particulier peut être accordé a des personnes ${ }^{34}$ ou à des territoires ${ }^{35}$ en fonction de critères autres que ceux prohibés par l'article 1er de la Constitution ;

En revanche, conformément à la logique de l'assimilation, on observera que les deux propositions de loi portant statut de ces populations, l'une déposée à l'Assemblée Nationale en mai 1972, l'autre en 1984, ne furent jamais votées ${ }^{36}$.

Dans les premières années de la décentralisation, la tradition assimilatrice l'emporte clairement sur le respect des particularismes. En estil de même avec les collectivités territoriales?

\section{B - Les pratiques des collectivités territoriales}

Un triple constat s'impose : le département apparaît comme l'interlocuteur privilégie des autorités coutumières, la région comme la garante de l'identité culturelle et les communes comme des collectivités de proximité.

\section{1) Le Département, interlocuteur privilégié des autorités} coutumières

La départementalisation de la Guyane par la loi de 1946 a laissé subsister l'organisation traditionnelle aux côtés des autorités de la

33 CE Ass., 13/7/1962, Conseil national de l'ordre des médecins, Rec. 479; RD publ. 1962.739, concl. Braibant. Sect. 10/5/1974, Denoyez et Chorques, Rec. 274 ; AJ 1974.298, chr. Franc et Boyon.

${ }^{34}$ Loi $\mathrm{n}^{\circ}$ 97-940 du 16 novembre 1997 relative au développement d'activités pour l'emploi des jeunes, J.O. du 17 octobre 1997, p. 15076.

${ }^{35}$ Loi n ${ }^{\circ} 89-486$ du 10 juillet 1989 d'orientation sur l'éducation, J.O. du 14 juillet 1989, p. 8860.

${ }^{36}$ Cf. la présentation et le commentaire du texte de l'avant projet de 1984, «Proposition de Loi portant statut des populations amérindiennes et noirs réfugiées de Guyane Française », in : J.P. Martres, J. Larrieu. Coutumes et droit en Guyane. Paris : Economica, 1993. 
République, Chefs et Grand chef coutumiers pour les Amérindiens ${ }^{37}$, Gran Man, capitaines et lieutenants pour les Bushinenge, désignés selon les usages reconnus par la coutume. Ces usages, par définition ne sont pas codifiés par écrit ; ils sont donc susceptibles de varier dans le temps mais aussi dans l'espace. On notera, par exemple, l'émergence du processus électif, circonscrit pour l'heure à quelques villages amérindiens (exemple de Balaté), alors que la désignation coutumière relève généralement du consensus et de l'hérédité.

La désignation coutumière est en principe suivie d'un processus administratif d'agrément par l'autorité administrative ${ }^{38}$. La portée de cet acte n'est pas négligeable : d'une part, il constate l'accomplissement des procédures coutumières de nomination (leur existence mais pas leur régularité, car cela reviendrait a s'immiscer dans la sphère coutumière); d'autre part, il conditionne le bénéfice d'indemnités imputées sur le budget départemental.

Jusqu'en 1982, c'est le préfet qui, en sa double qualité d'exécutif du département et de représentant de l'Etat, prend des décisions portant agrément des nominations coutumières. Avec le transfert du pouvoir exécutif du préfet vers le président du conseil général, cette compétence relève désormais de la collectivité départementale.

Toutefois la question de l'étendue du pouvoir d'agrément reste posée. C'est à cette question que devait répondre la collectivité, à la suite du litige survenu en 2007 au village de Balaté ${ }^{39}$, du fait de la désignation par une partie de la population d'un nouveau chef coutumier au lieu et place du chef coutumier agréé par la collectivité. Face à cette situation inédite, la collectivité a adopté une position médiane : elle a maintenu le versement des

\footnotetext{
${ }^{37}$ Cf. Collomb, op. cit., p. 122 : «Ce personnage (le chef coutumier chez les Kali'na) joue toujours un rôle essentiel, comme intermédiaire avec les institutions extérieures et comme acteur central de la vie communautaire au sein des villages».

${ }^{38}$ En 1984, interrogé par le président du Conseil général sur la procédure de nomination des chefs coutumiers, le préfet de la Guyane précise que « la nomination de Grands 'Mans, capitaines et lieutenants parmi les autorités coutumières des populations tribales a été décidée à l'époque du territoire de l'Inini afin de permettre à l'administration de disposer de représentants chargés d'assurer l'application de mesures diverses et de jouer en tant que de besoin le rôle d'auxiliaire de justice ».

${ }^{39}$ Commune de Saint-Laurent du Maroni.
} 
indemnités au chef coutumier agréé et à la suite de la démission de ce dernier, elle a agréé le chef coutumier nouvellement élu. Cette compétence connait ainsi une limite essentielle, celle tirée du respect du fonctionnement des institutions coutumières.

Une autre interrogation porte sur le texte donnant compétence à la collectivité en matière d'agrément des autorités coutumières. A vrai dire, il semble qu'aucune disposition, ni législative ni réglementaire, ne donne compétence en ce domaine à la collectivité. Il faut donc en conclure que ce pouvoir d'habilitation s'exerce en dehors de tout cadre légal et qu'en particulier les indemnités versées aux chefs coutumiers ne résultent d'aucune obligation juridique. Consenties discrétionnairement par la collectivité, elles apparaissent comme la contrepartie d'une activité dont les contours restent mal définis.

On observera par ailleurs que la position du département n'est pas linéaire : elle dépend de la sensibilité politique de l'exécutif territorial, du rôle reconnu aux autorités coutumières ou encore de la représentativité politique des populations concernées. Entre 1979 et 2003, plusieurs délibérations traitent des relations entre le département et les autorités coutumières. On citera principalement ici la délibération $n^{\circ} 44-92$ du 26 juin 1992 indiquant que le conseil général «se réserve en fonction des situations, la faculté de se prononcer sur la création de nouveaux postes de chefs coutumiers ${ }^{40}$. Douze ans plus tard, cette stratégie continue à régir l'action de la collectivité à l'égard des autorités coutumières. On le voit donc, dès le début de la décentralisation, la spécificité de l'organisation coutumière est prise en compte par le département ; par la suite, celui-ci fera preuve d'un intérêt croissant envers les autorités coutumières ${ }^{41}$. Aujourd'hui, l'intérêt de la collectivité pour l'institution coutumière se manifeste sous diverses formes : délégation au $1^{\text {er }}$ vice-président pour les relations département/autorités coutumières, réflexion sur l'opportunité de mettre en place de nouvelles procédures, de revaloriser le montant des indemnités,

\footnotetext{
${ }^{40} 42$ chefs coutumiers (dont une femme, Mme Kouyouri Cécile, «capitaine du village de Bellevue, commune d'Iracoubo) sont pris en charge par la collectivité ; celle-ci leur verse une indemnité correspondant à 50\% (Gran Man) et 30\% du SMIC (capitaines) et attribue à chacune des autorités coutumières agréées, une tenue officielle et deux tenues de travail.

${ }^{41}$ En assistant, par exemple, aux cérémonies d'investiture de chefs coutumiers.
} 
d'élargir la représentativité coutumière, projet d'organisation d'un séminaire sur les autorités coutumières.

\section{2) La Région, garante de l'identité culturelle}

La loi du 2 mars $1982^{42}$ fait de la préservation de l'identité une compétence régionale ; la loi du 2 août $1984^{43}$ reconnaît aux régions d'outremer des compétences spéciales dans les domaines de l'éducation, de la recherche, de la culture et de la communication. Un rôle prééminent dans la valorisation de son identité culturelle est ainsi reconnu à la région. D'abord les langues et cultures régionales peuvent faire l'objet d'activités éducatives et culturelles complémentaires. Ensuite, un programme culturel régional portant notamment sur les langues régionales, la littérature, les arts plastiques musicaux et cinématographiques est établi par le conseil régional. Enfin, la région assure le développement du patrimoine spécifique dont la conservation est définie et programmée dans le cadre d'un collège régional du patrimoine.

Ces compétences sont à l'origine d'un grand nombre de manifestations et d'initiatives en faveur des cultures locales. Il en est ainsi de la restauration et de la mise en valeur de sites historiques, de l'inventaire du patrimoine architectural et mobilier, des recherches et des cartes archéologiques, des subventions diverses aux associations culturelles, de la création d'équipements culturels (Musée des cultures guyanaises ${ }^{44}$, Office culturel de la Région Guyane, Ecole nationale de musique et de danse Edgard Nibul...). Surtout, elles ont permis l'enseignement dans les établissements scolaires et universitaires des langues et cultures régionales. Parallèlement, la région s'est engagée dans une démarche de promotion et de valorisation des langues et cultures régionales ${ }^{45}$. On pourrait donc croire que se trouve ainsi prise en compte la diversité culturelle de la Guyane. En fait, au cours de cette période, seules la langue et la culture créoles font l'objet

\footnotetext{
42 J.O. du 3 mars 1982, p. 730.

43 J.O. du 3 août 1984.

${ }^{44}$ Dont le Président est un Bushinengue.

45 Organisation de colloques, de séminaires par le Conseil de la culture, de l'éducation et de l'environnement régional, organe consultatif placé auprès du Conseil régional et qui est spécifique aux régions d'outre-mer.
} 
d'une stratégie de valorisation : il a fallu attendre 2006 pour que les langues amérindiennes et bushinenge fassent leur entrée à l'Université des Antilles et de la Guyane.

\section{3) Les communes, collectivités de proximité}

Les communes développent également des initiatives à l'égard des populations coutumières. Sur le littoral ${ }^{46}$, celles-ci concernent surtout la promotion des cultures régionales et le soutien financier aux associations représentatives de ces communautés. Dans quelques communes (Kourou, Mana, Iracoubo, Sinnamary), la présence au sein des conseils municipaux d'élus issus de ces communautés contribue à formaliser les relations avec la population concernée $e^{47}$ de telle sorte que la prise en compte des particularismes va parfois jusqu'à la mise en place d'un conseil consultatif (Saint-Laurent).

A l'intérieur de la Guyane ${ }^{48}$, les particularismes coutumiers amérindiens et bushinenge subsistent encore, favorisés, sans doute, par l'isolement géographique et l'homogénéité territoriale des communes concernées. Dès lors, pour l'institution communale, il s'agit surtout de concilier coutumes, traditions, et droit positif français ${ }^{49}$.

\footnotetext{
${ }^{46}$ Amérindiens et Bushinenge vivent principalement dans les communes de Saint-Laurent, Awala, Mana, Kourou, Cayenne ; on les trouve également près d' Yracoubo, au bord de la route qui mène à Saint-Laurent, entre Cayenne et Régina ; Richard et Sally. Price, op. cit. p.83.

${ }^{47}$ Arrêtés municipaux donnant délégation à un conseiller municipal pour les relations avec les autorités coutumières.

${ }^{48}$ Apatou, Grand-Santi, Papaichton, Camopi, Maripasoula, Saul.

${ }^{49}$ Les règles coutumières concernent le droit des personnes, les droits fonciers et les modes de règlement des litiges. Sur ces questions, cf. J.P. Martres, J. Larrieu, op. cit. Juridiquement se pose la question de la prise en compte de la coutume par le droit positif. Dans un pays de droit écrit, la coutume ne constitue pas une source de droit, toutefois dans la mesure où elle est compatible avec la légalité (Constitution, Traités, lois, ordonnances, principes généraux du droit, actes administratifs) l'administration ou le juge peuvent s'y référer ; Cf. l'exemple cité par un magistrat du TGI de Cayenne concernant les coutumes relatives au retour des morts ; D. Peyrat, «Le droit comme ressource », Droit et Cultures, 37(1), 1999, p. 155. En revanche en cas de conflit entre la règle coutumière et le droit positif, la hiérarchie des normes s'impose. Ce principe est rappelé par le Conseil d'Etat dans un arrêt d' Assemblée du 6 juin 1997, Aquarone. En l'espèce le juge précise qu'en cas de conflit entre la coutume
} 
Des développements qui précèdent, il ressort que les collectivités ont établi des relations avec les institutions coutumières dans des contextes juridiques diversifiés. Ces relations ont pourtant ceci de commun qu'elles se développent à l'initiative des collectivités qui décident de l'opportunité ou non de recueillir l'avis des communautés traditionnelles. Elles témoignent surtout de leur volonté de prendre en compte les particularismes coutumiers.

Aujourd'hui, le paysage constitutionnel a changé ; la réforme de 2003 comporte des dispositions favorables à l'expression des particularismes identitaires. Certes les Amérindiens et les Bushinenge de Guyane ne bénéficient pas d'une reconnaissance expresse au niveau constitutionnel ou législatif; ils font néanmoins l'objet de déclarations, de textes ou d'initiatives qui témoignent d'une réelle évolution de la part des pouvoirs publics. Dans ce contexte, il est sans doute permis de parler de reconnaissance explicite.

\section{II - La reconnaissance explicite des particularismes coutumiers}

Sur le plan international, les populations amérindiennes et bushiningue jouissent incontestablement d'une certaine reconnaissance d'un point de vue politique et symbolique. Au plan interne, le législateur prend désormais en compte leurs réalités spécifiques alors qu'au niveau local de nouvelles perspectives semblent se dégager en leur faveur.

\section{A - Vers une reconnaissance internationale ?}

Comme on le sait, la France rappelle régulièrement, dans les instances internationales, qu'elle ne reconnaît pas l'existence sur son sol de minorités ou d'autochtones ${ }^{50}$. Cette position a-t-elle évolué ? On est tenté de le croire à la lecture de la déclaration du représentant français lors de l'adoption par l'Assemblée générale des Nations Unies de la Déclaration des

internationale et la loi interne, aucun principe constitutionnel ne permet au juge de faire prévaloir la coutume sur la loi.

${ }^{50}$ En 1989 par exemple, un de ses représentants affirme que la France n'est « constituée que de citoyens et n'a donc pas de populations autochtones ». Cit. Par F. Morin, « Vers une déclaration universelle des droits des peuples autochtones », dans H. Giordan (dir.). Les minorités en Europe. Paris : Kimé, 1992. p. 493-507. 
droits des peuples autochtones ${ }^{51}$ : «Au niveau national, la France, directement concernée par les populations autochtones de ses collectivités territoriales d'outre-mer, conduit les programmes de soutien à leur développement économique et social dans un cadre adapté aux spécificités de ces populations, ainsi qu'à leur expression culturelle. C'est à ce titre que la France a soutenu l'ensemble des processus engagés au niveau multilatéral et apporté en particulier un appui financier à la Décennie internationale des populations autochtones du monde ». Le représentant de la France précise ensuite la conception française de la protection des droits de l'homme en ajoutant: «Pour la France, en vertu du principe d'indivisibilité de la République et conformément au principe fondamental d'égalité et son corollaire, le principe de non discrimination, des droits collectifs ne peut prévaloir sur les droits individuels. Un traitement particulier peut cependant être accordé à des populations autochtones sur une base territoriale. Le droit à l'autodétermination, tout comme les consultations et référendums locaux, évoqués dans les articles 3, 4, 19, 20 et 30 de la Déclaration s'exercent conformément aux normes constitutionnelles nationales, comme le prévoit l'article 46 de la présente Déclaration. Enfin, l'article 36 concernant le droit

\footnotetext{
${ }^{51}$ La Déclaration a été adoptée, après 20 ans de négociations, par l'Assemblée Générale de Nations Unies le 13/9/2007 par 143 voix pour, 4 voix contre (Etats-Unis, Canada, Australie, Nouvelle-Zélande), 11 abstentions. Elle comporte 46 articles, précédés d'un préambule qui affirme « que les peuples autochtones sont égaux à tous les autres peuples en dignité et en droits, tous en reconnaissant le droit de tous les individus et de tous les groupes à la différence, à s'estimer différents et n'être respectés en tant que tels ».

L'article 3 pose le principe de leur autodétermination : " les peuples autochtones ont le droit à l'autodétermination. En vertu de ce droit, ils déterminent librement leur statut politique et assurent librement leur développement économique, social et culturel, ainsi que leurs systèmes juridiques ». Les articles 13 à 15 concernent la pérennité des coutumes et cultures traditionnelles ainsi que l'enseignement. L'article 28 pose le principe du droit à réparation : «Les peuples autochtones ont droit à réparation, par le biais, notamment, de la restitution ou, lorsque cela n'est pas possible, d'une indemnisation juste, correcte et équitable pour les terres, territoires et ressources qu'ils possédaient traditionnellement ou occupaient ou utilisaient et qui ont été confisqués, pris, occupés, exploités ou dégradés sans leur consentement préalable, donné librement et en connaissance de cause... Sauf si les peuples concernés en décident librement d'une autre façon, l'indemnisation se fait sous forme de terres, de territoires et de ressources équivalents par leur qualité, leur étendue et leur régime juridique, ou d'une indemnité pécuniaire ou de toute autre réparation appropriée ». L'article 29 se réfère aux droits territoriaux et à l'environnement. L'article 44 précise enfin que tous ces droits s'appliquent également aux hommes et aux femmes.
} 
des populations autochtones à entretenir des relations internationales se lit dans le cadre des normes constitutionnelles dans ce domaine $»^{52}$.

Cette déclaration appelle plusieurs observations. On notera d'abord qu'à la négation juridique de l'existence des autochtones succède une reconnaissance explicite de leur existence. Cependant une question se pose : que recouvre la notion de collectivités territoriales d'outre-mer, lorsque l'on sait que les départements (DOM) les régions d'outre-mer (ROM), les collectivités d'outre-mer (COM) et les collectivités à statut particulier ${ }^{53}$ sont les seules catégories mentionnées par la Constitution?

On relèvera ensuite que la Déclaration mentionne les populations autochtones. L'autochtonie est un concept récent élaboré par le droit international qui trouve son origine dans le rapport $\mathrm{COBO}^{54}$. La convention $\mathrm{n}^{\circ} 169^{55}$ de l'Organisation Internationale du Travail (OIT) ${ }^{56}$ substitue la notion de peuple indigène à celle de peuple autochtone. A défaut d'une définition précise, ces textes énumèrent les éléments d'identification de cette notion : conditions sociales et économiques moins avancées que celles de la société dominante ; existence d'une culture, de coutumes et traditions spécifiques ou d'une législation particulière ; conscience de ces particularismes et volonté de les préserver ; origine inscrite dans une filiation vis-à-vis des populations qui habitaient le pays à l'époque de la conquête et de la colonisation ${ }^{57}$. S'interrogeant sur les populations d'outre-mer susceptibles de relever de la catégorie d'autochtones, le professeur Rouland identifie les Canaques de la Nouvelle Calédonie, les Amérindiens de Guyane, les Polynésiens, les Wallisiens et les Mahorais. Au regard des

\footnotetext{
${ }^{52}$ Nations Unies, A/61/PV.108.

${ }^{53}$ Saint-Pierre et Miquelon, Mayotte, Nouvelle-Calédonie.

${ }^{54}$ J.R. Martinez Cobo, » Etude du problème de la discrimination à l'encontre des populations autochtones, » E/CN.4/Sub.2/1986/7/Add.4. Référence citée par N. Rouland, S. Pierré-Caps, J. Poumarède. Droit des minorités et des peuples autochtones, précité, p. 398 .

${ }_{55}$ Concernant les peuples indigènes et tribaux dans les pays indépendants.

56 Adoptée en 1989 et entrée en vigueur en septembre 1991. Bulletin officiel du Bureau International du Travail, vol. L22/série A/n² , Genève, 1989.

${ }^{57}$ Observons que seuls les Amérindiens remplissent l'ensemble des conditions. R. et S. Price rappellent en effet «qu'aucun des peuples marrons n'est originaire de la Guyane. Tous viennent du Suriname, mais chaque groupe est arrivé à un moment historique donné, dans des circonstances particulières, héritant ainsi de statuts et de problèmes spécifiques. ", op. cit. p. 7.
} 
critères de la Convention de l'OIT, on peut se demander si les Bushinenge ne devraient pas être inclus dans cette liste.

On notera par ailleurs, la possibilité explicitement reconnue de prendre en compte les particularités autochtones sur une base territoriale et la réaffirmation de la tradition juridique française peu favorable aux thématiques du droit à la différence et à l'autodétermination des populations autochtones ou non autochtones .

Il reste enfin à préciser que la Déclaration en elle-même est dépourvue de toute portée juridique contraignante. Pour autant, sa portée politique et symbolique ne doit pas être minorée dans la mesure où ses dispositions fondent souvent les revendications des Amérindiens .

Quoi qu'il en soit, il n'est pas interdit de penser que les récentes initiatives prises par le législateur français en faveur des populations amérindiennes et noires-marrons s'inspirent des instruments internationaux de protection des droits des peuples autochtones et indigènes.

\section{B - La reconnaissance législative}

Deux lois marquent une étape importante dans la prise en compte des particularismes coutumiers : la loi n²006-436 du 14 avril 2006 relative aux parcs nationaux, aux parcs naturels marins et aux parcs naturels régionaux qui crée le Parc Amazonien en Guyane ${ }^{58}$ et la loi du 21 février 2007 portant dispositions statutaires et institutionnelles relatives à l'Outremer qui institue un conseil consultatif des populations amérindiennes et bushinenge.

Pour le législateur, il s'agit tout à la fois d'assurer une meilleure représentation des populations coutumières dans la gestion locale, de valoriser des originalités qui présentent un intérêt artistique et scientifique et de garantir une meilleure protection des intérêts spécifiques de ces populations.

\footnotetext{
${ }^{58}$ J.O. du 15 avril 2006, p. 5682, art. L331-15-1à L 331-15-7 du Code de l'Environnement.
} 


\section{1) Le Parc national dénommé Parc amazonien de Guyane}

La création du parc par le décret n ${ }^{\circ} 2007-266$ du 27 février $2007^{59}$ est l'ultime étape d'un processus qui a débuté en 1992, à la veille du Sommet de Rio $^{60}$. Les causes de cette lente gestation sont connues : d'abord les incertitudes quant aux priorités du parc, s'agissant de la protection de la faune, de la flore et de la forêt tropicale ou de la protection des populations menacées dans leur modes de vie traditionnel face aux objectifs économiques. Ensuite, l'importance et la complexité des enjeux ont contribué à ralentir le processus. C'est le cas pour le périmètre d'interdiction de l'exploitation aurifère, de l'évolution des modes de vie traditionnels autour de la chasse et de l'agriculture de subsistance, de la place du tourisme et de l'écodéveloppement. Enfin, la question, d'une part, des limites du parc, et d'autre part de la délimitation entre le cœur du parc (ancienne zone centrale protégée) et l'aire d'adhésion, a également joué un rôle.

Finalement, à l'issue d'une longue négociation entre l'Etat, les collectivités territoriales et les autorités coutumières, la loi du 14 avril 2006 crée le Parc Amazonien en Guyane ${ }^{61}$. Le décret du 27 février 2007 classe la majeure partie de l'intérieur de la Guyane en parc national ${ }^{62}$. Si le parc est incontestablement une structure étatique ${ }^{63}$, on observe que les représentants des collectivités territoriales (13 sièges) ainsi que les personnalités «à

\footnotetext{
${ }^{59}$ J.O. du 28 février 2007.

60 Protocole d'accord signé entre l'Etat et les Conseil régional et Général de Guyane prévoyant la création d'une mission d'étude financée par l'Etat, et d'un comité de pilotage associant élus locaux, fonctionnaires et personnalités scientifiques.

61 Avec notamment pour mission «de contribuer au développement des communautés d'habitants qui tirent traditionnellement leurs moyens de subsistance de la forêt, en prenant en compte leur mode de vie traditionnel. », art. L331-15-5.

62 Communes de Camopi, Maripasoula, Papaîchton, Saint-Elie et Saul soit $20000 \mathrm{~km}^{2}$ abritant 5300 habitants. On observera avec intérêt que le périmètre du Parc correspond, en partie, à celui du territoire de l'Inini. Hier, il s'agissait de protéger des communautés «en voie de disparition ». Aujourd'hui, il s'agit « d'assurer le bien-être des populations du Parc en prônant une reconnaissance de leur identité culturelle »... de maintenir et valoriser les coutumes et la diversité culturelle tout en ne freinant pas l'accès aux biens de consommation ». Programme de développement rural régional de la Guyane, 10 février 2007. Le changement d'orientation est manifeste.

${ }^{63}$ Il s'agit d'un Etablissement public national à caractère administratif dont le directeur est nommé par arrêté ministériel ; le préfet est commissaire du gouvernement auprès du conseil d'administration.
} 
compétence locale» ou «nationale » (16 sièges) occupent une place importante au sein de l'organe décisionnel. Mais l'originalité se situe ailleurs : elle résulte de la prise en compte du fait coutumier. Ainsi, «les communautés d'habitants qui tirent traditionnellement leurs moyens de subsistance de la forêt », concept apparu dans le décret de 1987, bénéficient de dispositions particulières (articles 19 à 22 du décret) ${ }^{64}$ permettant de prendre en compte les modes de vie traditionnels, notamment les pratiques cultuelles de ces communautés ${ }^{65}$. De même, siègent, au sein du conseil d'administration, cinq représentants des autorités coutumières désignés par le Gran-Man concerné ou à défaut par l'assemblée des capitaines et chefs de famille du territoire réunie par le maire de la commune concernée (art. 28). Une telle disposition appelle deux observations : d'une part, elle témoigne de l'évolution de la conception française de l'unité républicaine, un texte officiel reconnaissant désormais l'existence de la coutume ${ }^{66}$; d'autre part, elle semble résulter d'une double stratégie : celle de l'Etat, qui, confronté aux revendications des autorités coutumières, organise leur participation aux décisions les concernant et celle des autorités coutumières qui, pour obtenir satisfaction, utilisent la coutume comme «enjeu de pouvoir ${ }^{67}$.

\footnotetext{
${ }^{64}$ L'art. 22 du décret mentionne également les droits d'usage collectifs reconnus à ces communautés d'habitants.

${ }^{65}$ Ces communautés (dont le statut est associatif) bénéficient de droits d'usages collectifs pour la pratique de la chasse, de la pêche et de toute activité nécessaire à leur subsistance. Art. L 331-15-3 du C.E.

${ }^{66}$ A vrai dire, la volonté d'associer les autorités coutumières aux décisions les concernant n'est pas nouvelle. En témoigne le décret $n^{\circ}$ 98-165 du 13/3/1998 relatif à la création de la réserve naturelle de l'Amana sur le territoire des communes de Mana et Awala-Yalimapo ; ses dispositions prévoient que des représentants des autorités coutumières siègent au sein du comité consultatif de gestion de la réserve naturelle ( celle-ci constitue un site de ponte d'importance mondiale pour les tortues luths et vertes ). Une étape est franchie en 2007 : d'une part la reconnaissance légale du fait coutumier se situe à un rang supérieur dans la hiérarchie des normes, d'autre part la représentation coutumière dispose désormais de pouvoirs décisionnels.

${ }^{67}$ N. Rouland, op., cit., p. 532.
} 


\section{2) Le Conseil Consultatif des populations amérindiennes et bushinenge de Guyane $e^{68}$}

Sa création par la loi $n^{\circ} 2007-224$ du 21 février $2007^{69}$ à la suite d'un amendement du sénateur de Guyane, Georges Othily, constitue une novation intéressante $^{70}$. Ce Conseil est conçu comme un outil d'aide à la décision, chargé d'éclairer les collectivités départementale et régionale sur « tout projet ou proposition de délibération emportant des conséquences sur l'environnement, le cadre de vie et les activités culturelles des populations amérindiennes et bushinenge. » Certes ces compétences sont consultatives et circonscrites à quelques domaines limités, mais il s'agit là d'enjeux écologiques et humains majeurs pour la région ${ }^{71}$.

Sans doute, sa saisine par l'Etat et les collectivités (départementale et régionale) reste facultative, mais il n'est pas interdit de penser que, pour accroître la légitimité de leurs décisions politiques, les autorités concernées s'attacheront à recueillir systématiquement l'avis du conseil.

En revanche, deux mécanismes peuvent permettre à la nouvelle instance de jouer un rôle accru dans le futur : en premier lieu, la loi dote le conseil d'un pouvoir d'auto-saisine à l'égard de toutes questions entrant dans le champ de compétences de la région ou du département et intéressant

\footnotetext{
${ }^{68} \mathrm{Y}$ a-t-il une différence entre les termes bushinengue et noirs-marrons ? Dans leur ouvrage consacré aux marrons, R et S. Price observent qu' «Aujourd'hui en Guyane Française, la façon de désigner ces peuples dans leur ensemble se teinte d'une forte connotation politique. Les élus Aluku au Conseil régional et au Conseil général préconisent le terme bushinenge et rejettent les termes marrons et noir-marron qu'ils estiment relever du vocabulaire des européens. Les Saramaka, qui sont plus importants en nombre mais ne détiennent pas de sièges dans les assemblées délibérantes, n'apprécient pas le vocable «bushinenge » qui appartient à la langue des Aluku et de leurs voisins. Nous utilisons le terme «Marrons parce qu'il est à la fois neutre en regard des rivalités entre ces groupes et porteur d'une connotation d' héroisme dans les luttes pour la liberté », op. cit. 7.

${ }^{69}$ Portant dispositions statutaires et institutionnelles relatives à l'Outre-mer, J.O. du 22 février 2007 , p. 3220. Cf. également le décret n ${ }^{\circ} 2008-562$ du 17/6/08 relatif à la composition, à l'organisation et au fonctionnement du conseil consultatif des populations amérindiennes et bushinengue de Guyane, J.O., 18/6/08.

70 Il est composé de 20 membres (16 représentants d'organismes et associations, 4 personnalités qualifiées) désignés pour 6 ans renouvelables.

${ }^{71}$ Le thème de l'environnement fait l'objet d'une attention particulière des institutions européennes (Parlement européen, Conseil des ministres, Commission européenne) et internationales (ONU).
} 
directement les populations amérindiennes et bushinenge. A ce titre, il appartiendra au Conseil d'appeler l'attention des pouvoirs publics sur les réformes législatives et réglementaires qui lui paraissent conformes à l'intérêt des populations coutumières.

En second lieu, la possibilité de tenir des séances communes avec le Conseil de la culture, de l'éducation et de l'environnement et le Conseil économique et social peut lui permettre de s'assurer l'appui de personnalités siégeant dans les conseils consultatifs de la collectivité régionale.

On notera, par ailleurs, que la représentativité des populations s'effectue au travers d'organismes et d'associations représentatifs des populations amérindiennes et bushinenge ${ }^{72}$. L'originalité de cette représentation mérite d'être soulignée dans la mesure où les autorités coutumières semblaient jusqu'alors seules susceptibles d'incarner la légitimité. Or dans un système démocratique, l'organisation et la représentation politique des groupes s'expriment à travers différents mécanismes dont l'association constitue la forme la plus répandue. Dans ces conditions, il s'agit clairement, pour l'Etat, de tenir compte de l'importance croissante des associations amérindiennes et bushinenge dans la vie sociale guyanaise.

On relèvera enfin que l'Etat est omniprésent : il désigne les personnalités qualifiées siégeant au Conseil. Il arrête la liste des organismes et associations représentatifs des populations amérindiennes et bushinenge et constate leur désignation. Il peut assister aux réunions du conseil consultatif et le cas échéant être entendu. Il assure le secrétariat du Conseil. Il publie au recueil des actes administratifs de la préfecture les avis et délibérations adoptés par le Conseil.

Quoi qu'il en soit, la création d'un conseil consultatif constitue une avancée dont la portée peut faire l'objet d'appréciations diverses. Il n'en reste pas moins qu'il apparaît comme un forum potentiel pour les communautés concernées ${ }^{73}$.

72 Art. D. 4436 résultant du Décret 2008-562 du 17/6/08, J.O. 18 juin 2008, p. 9863.

${ }^{73}$ A noter que les avis du Conseil mentionnent les positions des minorités (art. D. 4436-5 du Code Général des Collectivités Territoriales). 
Faut-il aller plus loin ? C'est l'objectif que se fixe le projet d'évolution institutionnelle porté par les collectivités départementale et régionale de Guyane.

\section{C - Les perspectives locales}

Le document adopté par le Congrès des élus départementaux et régionaux de la Guyane en $1999^{74}$ marque une rupture. Il prévoit la création d'un conseil consultatif des autorités coutumières dans le cadre d'une collectivité nouvelle ${ }^{75}$.

L'on se souvient encore du débat qui a opposé en 2002-03 les tenants du statut de DOM (l'article 73) aux partisans de l'autonomie interne avec l'article 74. Ce débat n'est pas nouveau: dans un article publié en 1996, T. Michalon a montré que le statut de DOM constituait pour les populations autochtone et bushinengue « une impasse juridique ${ }^{76}$.

Les nouvelles dispositions constitutionnelles sur l'outre mer introduites par la réforme du 28 mars 2003 modifient-elles les données juridiques du problème $?^{77}$ La réponse paraît a priori positive. Au titre des éléments favorables, on relèvera que la nouvelle rédaction de l'article 73 comporte deux innovations : si le principe d'assimilation législative demeure la règle applicable aux DOM, ce principe peut cependant faire l'objet de mesures d'adaptation tenant aux caractéristiques et contraintes particulières de ces collectivités, (art. 73, alinéa 1).

Surtout, le troisième alinéa de l'article 73 semble comporter une ouverture dans la mesure où " pour tenir compte de leurs spécificités, les départements et les régions d'outre-mer pourront, être habilités par la loi à fixer elles mêmes les règles applicables sur leur territoire, dans un nombre limité de matières pouvant relever de la loi ».

\footnotetext{
74 Congrès des élus départementaux et régionaux du 27/2/1999 approuvant le document d'orientation d'un pacte de développement pour la Guyane.

${ }^{75}$ Il n'a jamais été adopté.

${ }^{76}$ T. Michalon. «Les aspects institutionnels de l'avant projet de 1984 », Coutumes et Droit en Guyane, op. cit., p. 153-164.

77 La réforme consacre 9 articles à l'outre-mer; les DOM/ROM sont particulièrement concernés par les articles 72 à 74 .
} 
En réalité, ce pouvoir local est strictement encadré : d'une part l'alinéa 4 énumère une série de domaines exclus du champ de l'habilitation législative ; d'autre part, une loi organique peut compléter cette liste. Enfin le risque d'encourir la censure du juge constitutionnel demeure réel dès lors que la prise en compte des particularismes s'inscrira dans un schéma institutionnel particulier. On est donc fondé à se demander si le statut de l'article 73, même dans sa nouvelle formulation, ne reste pas pour les populations amérindiennes et bushinenge, « une impasse juridique ».

La reconnaissance des particularismes juridiques autochtones seraitelle mieux assurée dans le cadre de l'article 74 ? Jusqu'en 2003, celui-ci prévoyait que les Territoires d'Outre-Mer ont « une organisation particulière tenant compte de leurs intérêts propres dans l'ensemble des intérêts de la République. » Dans une décision de 1985, le Conseil Constitutionnel a précisé la notion d'organisation particulière : " elle ne s'applique pas uniquement aux institutions proprement dites telles que l'Assemblée territoriale et le Conseil de gouvernement...elle couvre ce que l'on pourrait appeler le régime administratif (et aussi l'organisation juridictionnelle) $»^{78}$.

Naturellement, la nouvelle formulation de l'article 74 issue de la réforme constitutionnelle de 2003 ne remet pas en cause cette interprétation jurisprudentielle.

On sait par ailleurs que l'article 75 permet aux habitants qui n'ont pas le statut civil de droit commun, soit de conserver leur statut personnel, soit d'y renoncer en faveur du premier ${ }^{79}$. Enfin l'exemple calédonien (Titre XIII de la Constitution) montre que les discriminations positives fondées sur l'origine ethnique sont toujours possibles à condition qu'elles soient autorisées par la Constitution ${ }^{80}$.

L'année 2008 marque la reprise des travaux sur l'évolution institutionnelle de la Guyane ${ }^{81}$. Le contexte local semble aujourd'hui plus

\footnotetext{
78196 DC, 197 DC, 8 et 23/8/85, Evolution de la Nouvelle-Calédonie, GDCC, $11^{\text {e }}$ éd., p. 624. L'exemple de Wallis et Futuna ou de Polynésie montre que les institutions locales créées dans ce cadre permettent de prendre en compte les particularismes coutumiers.

${ }^{79}$ L'article 75 est appliqué en Polynésie, Wallis et Futuna, Nouvelle-Calédonie, Mayotte.

${ }^{80}$ Décis. $n^{\circ}$ 99-410 DC du 15/3/99, Loi organique relative à la Nouvelle-Calédonie, J.O. du

21 mars 1999, p. 4234.

${ }^{81}$ Congrès des élus régionaux et départementaux du 20/6/2008.
} 
favorable aux revendications des Amérindiens et des Bushinenge, cette évolution étant due en partie à l'action de ces communautés dont les représentants manient de mieux en mieux le langage et les usages administratifs. Mais un autre élément y contribue fortement : la conviction que la cohésion sociale et l'intégration des populations amérindiennes et bushinenge passent désormais par la reconnaissance de leurs identités culturelles et non par leur négation.

\section{Conclusion}

En définitive l'affirmation du principe unitaire n'exclut pas la reconnaissance des particularismes ; l'objectif poursuivi consiste à la fois à élargir la représentativité de ces populations, assurer leur participation aux décisions les concernant et renforcer leur intégration dans la société guyanaise. En fonction des objectifs poursuivis par les autorités administratives, l'expression de ces particularismes prend des formes diverses : représentation coutumière, médiation, structure associative, plurilinguisme $^{82}$. Cependant deux constats s'imposent : d'une part, Amérindiens et Bushinengue ne constituent pas des groupes homogènes ni culturellement $^{83}$, ni juridiquement, un grand nombre d'entre eux ne maîtrisant pas le français et ne possédant pas la nationalité française ${ }^{84}$. Or comme le rappelle le Professeur Rouland «la citoyenneté est le vecteur juridique d'un partage de valeurs communes ${ }^{85}$.

D'autre part, les politiques mises en œuvre ne répondent pas aux attentes des Amérindiens et des Bushinengue, ces derniers revendiquant «le

\footnotetext{
${ }^{82}$ Observons que la question autochtone (au sens large incluant les noirs-marrons), fait désormais partie de l'agenda politique ainsi qu'en atteste l'atelier consacré aux langues autochtones organisé à Awala-Yalimapo, dans le cadre du Congrès annuel de l'Association des collectivités et des communes d'Outre-mer (semaine du 17/11 au 22/11/08).

${ }^{83}$ Cf. supra, introduction. Il faut cependant mentionner quelques initiatives en ce sens, en témoigne la création en 1993 d'un conseil des chefs coutumiers de Guyane ou l'organisation d'un Congrès des autorités coutumières autochtones de la Guyane (nov. 2008). Les solidarités sont plutôt transnationales : relations avec les Kali'na et les A kuku du Surinam et du Brésil.

${ }^{84}$ Price, op.cit, p. 93 ; D. Davy « Vannerie et vanniers, approche Ethnologique d'une activité artisanale en Guyane française », p. 34 et suivantes. Thèse soutenue le 26/11/2007, Université d'Orléans.

${ }^{85}$ N. Rouland, Droit des minorités et des peuples autochtones, Paris, PUF, 1996.
} 
droit de choisir leur statut politique et celui de leur territoire ; le droit à la propriété communautaire ainsi que la préférence en matière juridique accordée à l'usage du droit coutumier pour juger leurs délits ${ }^{86}$. De fait, certaines Constitutions se sont engagées dans cette voie, à l'exemple du Canada et des Etats-Unis qui reconnaissent un statut particulier au profit des peuples autochtones ou des minorités. Il en va de même dans un certain nombre d'autres d'Etats qui pourtant ne présentent pas la forme fédérale mais unitaire, tels la Grande-Bretagne, les Pays-Bas ou la Scandinavie. On observera enfin qu'en Amérique du Sud comme en Amérique Centrale, de nombreux Etats confèrent aux autochtones un véritable statut constitutionnel, à l'instar du Guyana, du Suriname, du Brésil, de la Bolivie, du Costa Rica, de l'Equateur, du Guatemala, du Honduras, du Mexique, du Panama, du Pérou, du Venezuela, du Nicaragua, du Paraguay et de l'Argentine ${ }^{87}$.

Aujourd'hui, la France privilégie la protection des droits individuels sur les droits collectifs revendiqués par les Amérindiens et les Bushinengue. Et demain ? Assistera-t-on à une prise en compte progressive des droits des communautés amérindiennes et bushinengue. Il est permis de le penser, tant

${ }^{86}$ Résolution du Parlement européen en date du 9/2/1994 sur les mesures internationales nécessaires à une protection effective des peuples indigènes, JOCE, ${ }^{\circ} \mathrm{C} 61$ du 18/2/94, p.169. Cf. également A. Tiouka, Coordonnateur délégué de la Fédération des Organisations Amérindiennes de Guyane (FOAG), «Ce qu'ils veulent (les peuples autochtones), c'est assurer la survie de leurs communautés, la protection de leurs droits en tant que peuples autochtones et la reconnaissance d'une forme d'autonomie ou d'autodétermination ». Conférence sur les droits collectifs des peuples autochtones : le cas des Amérindiens de Guyane Française, A. Tiouka, IESG, 2000.

87 A titre d'exemple, la Constitution de la République fédérative du Brésil (1998) dispose «Sont reconnus aux Indiens leur organisation sociale, leurs coutumes, leurs langues, leurs croyances et leurs traditions ainsi que leurs droits originels sur les terres qu'ils occupent traditionnellement; il incombe à l'Union de les démarquer (terres), de les protéger et de faire respecter tous leurs biens (Indiens) ». Art. 231.

La Constitution péruvienne (1993) prévoit le droit de propriété collective des terres et la personnalité juridique des communautés autochtones (art. 88) ; la reconnaissance de l'identité culturelle des communautés autochtones (art.89) ; la reconnaissance du droit coutumier et de la juridiction coutumière sur les terres des communautés autochtones (art. 149) ; le principe d'un quota de représentants autochtones au sein des conseils municipaux et régionaux (art. 191).

La Constitution du Guatemala (1985, modifiée en 1993) consacre la reconnaissance des différences culturelles des autochtones et leurs droits sur les terres traditionnellement occupées (art. 66 à 70); le droit à l'éducation bilingue (art. 76). 
il est vrai que la France apparaît de plus en plus isolée sur la scène européenne et internationale. 


\title{
RESUME
}

\section{Pouvoirs publics, populations amérindiennes et bushinenge en Guyane Française : quelles relations depuis la décentralisation (1982-2008)}

Comme les autres départements d'outre-mer, la Guyane a connu l'Ancien régime et l'esclavage et son peuplement actuel est en partie le résultat de la colonisation. Sa départementalisation en 1946 sl'inscrit dans une logique d'assimilation législative ; sa spécificité par rapport aux autres DOM tient au fait qu'elle comporte encore une population autochtone d'origine et des descendants d'esclaves révoltés dont les particularismes coutumiers subsistent et s'expriment essentiellement dans le droit des personnes et des relations privées ; si les particularismes territoriaux ne posent pas de problèmes quant au respect de l'article 1 de la Constitution, le droit français, depuis la Révolution, exclut toute reconnaissance officielle de différences entre les individus à raison de leur origine, de leur race ou de leur religion ; pourtant l'absence de reconnaissance dans l'ordre constitutionnel interne, du droit de ces populations à préserver leur identité n'empêche nullement la mise en oeuvre progressive d'une pratique réglementaire et législative particulariste.

\begin{abstract}
Authorities and populations of American Indians and bushinenge in French Guiana : what has become the relationship since the decentralization (1982-2008)

Like the other overseas departments, French Guiana has experienced the Ancien Régime and slavery, and its present population is partly a result of colonisation. Its conversion into a French overseas department in 1946 falls within the scope of a legislative assimilation process. Compared to other DOM (overseas departments) its specificity lies in the fact that it still comprises a native population as well as rebellious descendants of slaves, whose customary sense of identity subsists and is essentially expressed in the rights of people and private relations. The territorial sense of identity does
\end{abstract}


not pose problems as to the respect of article 1 of the Constitution, - since the Revolution, French law is excluding any kind of official recognition of differences between individuals on the grounds of their origin, race or religion. Yet the absence of recognition in the internal constitutional order of the right of these populations to preserve their identity does in no way prevent the progressive implementation of a statutory practice and specific legislature. 